\title{
Joint Modeling of Time to Diabetic Retinopathy and Change in Fasting Blood Sugar Among Type 2 Diabetic Patients in University of Gondar Comprehensive Specialized Hospital, Northwest Ethiopia
}

Sewnet Adem Kebede ( $\square$ sewnetme1@gmail.com )

University of Gondar College of Medicine and Health Sciences https://orcid.org/0000-0001-7750-7596

\section{Zemenu Tadesse Tessema}

University of Gondar College of Medicine and Health Sciences

Shitaye Alemu Balcha

University of Gondar College of Medicine and Health Sciences

Tadesse Awoke Ayele

University of Gondar College of Medicine and Health Sciences

\section{Research}

Keywords: Type 2 diabetes mellitus, Diabetic retinopathy, Fasting blood sugar

Posted Date: April 19th, 2021

DOI: https://doi.org/10.21203/rs.3.rs-422760/v1

License: (9) This work is licensed under a Creative Commons Attribution 4.0 International License.

Read Full License 
1 Joint modeling of time to diabetic retinopathy and change in fasting blood

2 sugar among type 2 diabetic patients in University of Gondar

3 comprehensive specialized hospital, Northwest Ethiopia

$4 \quad{ }^{1}$ Sewnet Adem Kebede*, ${ }^{1}$ Zemenu Tadesse Tessema, ${ }^{2}$ Shitaye Alemu Balcha, ${ }^{1}$ Tadesse

5 Awoke Ayele

$6 \quad{ }^{1}$ Department of Epidemiology and Biostatistics, Institute of Public Health, College of

7 Medicine and Health Sciences, University of Gondar, Gondar, Ethiopia

$8{ }^{2}$ Department of Internal Medicine, School of Medicine, College of Medicine and Health

9 Sciences, University of Gondar, Gondar, Ethiopia

10 *Corresponding Author's email address

11 SAK: $\underline{\text { sewnetme1@gmail.com }}$

13 Abstract

14 Background: Early identification of prognostic factors and close monitoring of the

15 biomarkers is used to monitor the occurrence or progression of diabetic retinopathy.

16 Therefore, this study aimed to assess change in fasting blood sugar level, time to diabetic

17 retinopathy and its predictors among type 2 diabetes patients in Ethiopia.

18 Methods: Institution based retrospective follow-up study was conducted at University of

19 Gondar Comprehensive Specialized Hospital. Linear mixed effect model and cox

20 proportional hazard models were fitted separately and later the two models were fitted

21 jointly using $\mathrm{R}$ software. Variables with $\mathrm{p}$-value $<0.05$ were considered as significant

22 predictors in the adjusted analysis.

23 Result: The incidence rate of diabetic retinopathy was 2 per 100-person year of observation

24 with median follow up time of 90.8 months (IQR: 63.4). The current value and rate of 
25 change in fasting blood sugar level were significant predictors of time to diabetic retinopathy $\quad(\mathrm{AHR}=1.35 ; 95 \% \quad \mathrm{CI}: \quad 1.12-1.63)$ and $\quad(\mathrm{AHR}=1.70 ; 95 \% \mathrm{CI}: 1.21-2.39)$

respectively. Hypertension (AHR=2.49; 95\%CI: 1.32-4.66), taking >1 antidiabetic oral agent $(\mathrm{AHR}=4.90 ; 95 \% \mathrm{CI}: 1.07-20.0)$ and more than 10 years duration $(\mathrm{AHR}=0.17,95 \%$ CI: 0.06-0.46) were predictors of time to diabetic retinopathy.

Conclusion: This study revealed that medication, duration, weight, current age, and time

31 have significant association with the progression change of fasting blood sugar. On the

32 other hand, hypertension, medication and duration were significant predictors of time to 33 diabetic retinopathy. The current value of fasting blood sugar and rate of fasting blood 34 sugar change were significantly associated with time to diabetic retinopathy.

35 Key words: Type 2 diabetes mellitus, Diabetic retinopathy, Fasting blood sugar

36

\section{Background}

38 Diabetes mellitus (DM) is a metabolic disorder of multiple etiology characterized by 39 chronic hyperglycemia with disturbances of carbohydrate, fat and protein metabolism 40 resulting from defects in insulin secretion, insulin action or both $(1,2)$.

41 Type 2 diabetes mellitus is the most common form and covers $90 \%$ of people with diabetes 42 around the world $(2,5,6)$. Type $2 \mathrm{DM}$ patients are more susceptible to various forms of 43 complications such as cardiovascular disease, neuropathy, nephropathy and eye disease, 44 leading to retinopathy and blindness $(4,5,7)$.

45 Diabetes mellitus has a long term effect on the retinal small blood vessel which cause 46 progressive development of the specific complications of retinopathy, resulting in vision 47 loss $(1,8)$. Diabetic retinopathy (DR) is the leading cause of vision loss in working age 48 adults (20 to 65 years) and approximately one in three people living with diabetes have 
some degree of DR and one in ten will develop a vision threatening form of the disease (5).

50 Globally, the number of people with DR will grow from 126.6 million in 2010 to 191.0

51 million by 2030, and it is estimated that the number with vision-threatening DR will

52 increase from 37.3 million to 56.3 million, if prompt action is not taken (9).

53 The magnitude of DR in Africa range from 30.2 to $31.6 \%$ and 7.0 to $62.4 \%$ in patients with

54 diabetes in population-based studies and clinic-based surveys respectively (10).A

55 population-based study in Kenya identified a prevalence of DR 35.9 in 277 people with

56 diabetes (11). In Ethiopia the magnitude of DR ranged from 5.0\% in northwest Ethiopia to

$5743.4 \%$ for T2DM patients in Southwest Ethiopia. Many studies done in Ethiopia showed

58 that the presence and severity of complications related to DR are steadily increasing (12).

59 Risk factors for diabetic retinopathy among diabetes patients includes gender, uncontrolled

$60 \mathrm{DM}$, longer duration of DM, presence of nephropathy and presence of hypertension were

61 documented in different studies (13-16).

62 Until an advanced stage DR is asymptomatic and is largely preventable through regular

63 retinal screening and prompt treatment before their visual deterioration. However,

64 screening for diabetic retinopathy is not performed as expected for diabetes patients in

65 resource limited area because it is not easily accessible and affordable.

66 So, monitoring the biomarkers is very essential to be aware of that metabolic abnormality,

67 poorly controlled blood sugar, which increases the likelihood of retinopathy occurring and

68 cause retinopathy to progress more quickly if it does occur and provides meaningful

69 prognostic information that can help to differentiate patients with regard complication and

70 lead the way to change other intervention. 
71 Previous studies conducted in Ethiopia used separate analysis which ignore the dependency and association between longitudinal FBS and time to diabetic retinopathy. Joint modeling

73 approach is preferred than separate survival model as it provides more efficient estimates

74 of the treatment effects on the time to diabetic retinopathy and longitudinal marker of FBS.

75 Hence, this study uses joint modeling approach to account for the dependence and 76 association of time to diabetic retinopathy and fasting blood sugar change.

\section{Methods}

\section{Study design and area}

79 An institutional-based retrospective follow-up study was conducted from January 2001 to 80 February 2016 among type 2 diabetes patients in University of Gondar Comprehensive 81 Specialized Hospital (UGCSH). UGCSH serves more than five million people of the North

82 Gondar zone and peoples of the neighboring zones. Around 24,862 numbers of people are 83 having chronic follow-up per year and among this 8,900 are DM patients.

\section{Source Population}

85 The source population were all type 2 diabetes patients having a follow up at UGCSH. All 86 newly diagnosed type 2 diabetes patients in UGCSH from January 2001 to February 2016

87 were included in the study. However, those whose date of initiation was not recorded, those 88 who had only one fasting blood sugar measurement and newly diagnosed patients who had 89 DR at the start of follow up were excluded.

\section{Sample size determination and sampling procedure}

91 The sample size was determined for the two processes; longitudinal and survival.

\section{Sample sized for longitudinal outcome}


93 The sample size was determined by considering the following statistical assumptions: two-

94 sided significance level $(\alpha=5 \%)$, power $80 \%$, number of repeated measurements $(m=7)$,

95 within subject correlation $(\rho=0.5)$ and effect size $(\delta=0.0384) \quad$ using

$96 \mathrm{n}=\frac{2\left(z_{\frac{\alpha}{2}}+z_{\beta}\right)^{2}(1+(m-1) \rho}{m \delta^{2}}(19)$. The effect size $(\delta)$ and number of repeated measurements $(\mathrm{m})$

97 for calculating the sample size in this study was taken from study in Debre Berhan referral

98 hospital, Ethiopia (20) and the calculated sample size was 466.

\section{Sample sized for survival outcome}

100 To estimate survival probability of patients we consider sex as exposure based on a research 101 conducted in Tikur Anbessa Hospital in Addis Ababa (21).Using STATA 14.1 by using 102 power analysis for $\log$ rank test and considering $\mathrm{Z}$ value at $95 \%$ confidence, Power $80 \%$ 103 and $10 \%$ incompleteness, the sample size was 435.

104 Finally, largest sample size (466) was selected. With 5\% contingency the final sample size 105 required for this study was 489. After the patients fulfill selection criteria, study subjects 106 were selected by using systematic random sampling technique.

\section{Variables}

108 The response variable for the survival sub model was time from the date of diagnosis until 109 the occurrence of either diabetic retinopathy or censoring measured in months, while the 110 response variable for longitudinal sub model was blood glucose level in terms of fasting 111 blood sugar using mill gram per deci liter $(\mathrm{mg} / \mathrm{dL})$ from treatment start (baseline) and 112 repeatedly measured every three months, were time varying endogenous covariate. Socio 113 demographic variables: baseline age (year), current age (year) and gender. Clinical 114 variables: blood glucose, lipid level profile, body mass index (BMI), albuminuria level, 
115 diabetic duration, treatment type and creatinine clearance Comorbidities: hypertension, neuropathy, nephropathy and anemia were independent variables.

\section{Variable definition}

118 For this study, Diabetic retinopathy, time to diabetic retinopathy, censored, poor glycemic 119 control and hypertension were defined as follows: Diabetic retinopathy: was established if 120 the subject has a minimum of one micro aneurysm in any field, or showing hemorrhages 121 (dot \& blot, or flame shaped), or maculopathy (with or without clinically significant edema 122 (15). Time to diabetic retinopathy is the time between the times of diagnosed as T2DM to 123 the development of diabetic retinopathy. Censored includes lost to follow up, death and

124 being event free at the end of the study. Hypertension diagnosed when SBP is greater and 125 equal to $140 \mathrm{mmHg}$ and DBP is greater and equal to 90. Poor glycemic control was 126 established if fasting blood sugar is greater or equal to $140 \mathrm{mg} / \mathrm{dL}$.

\section{Data collection and data quality control}

128 The study exclusively used secondary data and structured data extraction checklist. Four 129 trained professional working in chronic follow-up clinic were recruited for data collection. 130 Socio-demographic characteristics, baseline and follow up clinical and laboratory data 131 collected from patient cards. Height measured as standing height using standard height 132 scale for adults. Weight of the patients were recorded using analogue weighing scale. Blood 133 Pressure readings measured using mercurial sphygmomanometer. Diabetic retinopathy 134 identified based on history of Diabetes Mellitus and findings of fundoscopy. Diabetic 135 retinopathy grading in this study have been described based on diabetic retinopathy 136 screening to treatment guideline (22). A week before the actual data collection preliminary 137 review was done on similar area. The data extraction sheet was pre-test for consistency of 138 understanding, adequacy of instrument, time requirement to fill the check list of tools and 
completeness of data for charts. Necessary adjustment for the final data collection sheet

140 was made by excluding variables which is not found on charts like educational status and

141 history of smoking. Training on the objective of the study and how to retrieve records as

142 per data extraction sheet was given to data collectors and supervisors for two days before

143 data collection. The information formats cross checked with the source card on the spot and

144 regular supervision was done. The data was entered using Epi-info 7 and then export to

145 STATA 14.1 and R 3.4.3 statistical software's for further data cleaning and statistical 146 analysis.

\section{Statistical analysis}

148 Descriptive measures such as means, median, IQRs, percentages, frequencies and standard 149 deviations were used to describe the study population. The survival experience of the 150 patients was assessed using Kaplan-Meier survivor function. The log rank test was used to 151 compare the survival experiences among the different groups of subjects. Cox PH and three 152 parametric models (Weibull, Exponential and Log logistic) were fitted to identify the risk 153 factors. The best model was selected by using Akaike information criteria (AIC). 154 Accordingly, the Cox PH model was the preferred model to model time-to-diabetic 155 retinopathy among type $2 \mathrm{DM}$ patients in the study area. Schoenfeld residuals test and 156 graphical methods were used to check the Cox Proportional Hazard (PH) assumption before 157 fitting survival sub model. Goodness of fit of the model was assessed by using Cox-Snell 158 residual technique.

159 To account for the effect of an endogenous time-varying covariate (FBS) on the time to DR 160 the true unobserved value of fasting blood sugar in the survival model was used. The 161 trajectory FBS over time was approximately normally distributed. Exploratory analysis was 162 used to visualize the patterns of individual profiles plot and average evolution change 
graphically. Linear mixed effect model with random intercept only and both random intercept and slop was fitted. Linear random effect model with both intercept and slope was best model that appropriately predicts the mean change of FBS measurements over time. To estimate the effects of longitudinal fasting blood sugar change to the risks of diabetic retinopathy the complete true history of fasting blood sugar for each subject using linear mixed effect model constructed by considering effects of baseline covariate on fasting blood sugar evolution. Association parameter (alpha value) from fitted joint model was used to assess association between longitudinal biomarkers (fasting blood sugar) and time to diabetic retinopathy.

\section{Model specification}

\section{Linear mixed modeling}

Linear mixed models are a type of regression model that take into account both variation that is explained by the independent variables of interest and variation that is not explained by the independent variables of interest. This is due to the measurement taken from the same subject at different time points or the measurements taken from the same clusters are likely to be correlated. In this study before the joint modeling to have an appropriate longitudinal sub-model for the longitudinally measured fasting blood sugar the LMM were employed to identify the covariates that have significant effects on the mean change of FBS measurements over time. Therefore, the longitudinal data modeling was beginning with exploratory data analysis to determine the mean change of FBS measurement over time. To measure the effect of the longitudinal covariate to the risk for an event, we need to estimate the true unobserved value of the longitudinal covariate $m_{i}(t)$ and successfully reconstruct the complete longitudinal history $M_{i}(t)$ for each subject. To achieve this, we 
postulate a suitable mixed-effects model to describe the subject-specific time evolutions with this notation

$$
y_{i}(t)=m_{i}(t)+\varepsilon_{i}(t)
$$

189

$$
m_{i}(t)=x_{i}(t) \beta_{1}+z_{i}(t) b_{i}
$$

191 where we explicitly note that the design vectors $X_{i}(t)$ for the fixed effects $\beta$, and $Z_{i}(t)$ for

192 the random effects $b_{i}$, as well as the error terms $\varepsilon_{i}(t)$, are time dependent. We assume that

193 error terms are mutually independent, independent of the random effects, and normally

194 distributed with mean zero and variance $\sigma^{2}$.

\section{Survival sub modeling}

196 Survival models seek to explain how the hazard of an event occurring at a given time is

197 affected by covariates. Hazard function of survival model is used to explain the probability

198 that the event has occurred by duration t. This study considered semi-parametric survival 199 models to explain how the risk, or hazard, of diabetic retinopathy occurring at a given time 200 is affected by covariates in the study area. Cox proportional hazard model which expresses 201 the hazard of an event at time $t$ as:

$$
\lambda_{i}(t)=\lambda_{o}(t) \exp \left(W^{T} \gamma\right)
$$

203 Where $\mathrm{W}$ is the matrix of baseline covariates, $\gamma$ is the vector of parameters and the term

$204 \lambda_{o}$ is the baseline hazard where the effects of covariates are zero. 
206 Joint model consists of longitudinal and survival sub model. Joint model of longitudinal 207 and time-to-event data can effectively assess the impact that a longitudinal covariate, 208 measured with error, has on the time to an event of interest. In this study we assess the 209 predictive ability of a biomarker FBS on time to diabetic retinopathy. To introduce this 210 methodology, we will use the following notation to harmonize diverse approach.

211 In particular, we denote by $T_{i}{ }^{*}$ the true event time for the $i^{\text {th }}$ subject, $T_{i}$ the observed event 212 time, defined as the minimum of the potential censoring time $C_{i}$ and $T_{i}{ }^{*}$, and by $\delta_{i}=$ $213 I\left(T_{i}{ }^{*} \leq C_{i}\right)$ the event indicator. For the endogenous time-dependent covariate (FBS) we let $214 y_{i}(t)$ denote its observed value at time point $t$ for the $i^{\text {th }}$ subject. We should note that we 215 do not actually observe $y_{i}(t)$ for any time $t$, but rather only at the very specific occasions $216 t_{i j}$ at which measurements were taken. Thus, the observed longitudinal data consist of the 217 measurements

$218 y_{i j}=\left\{y_{i}\left(t_{i j}\right), j=1, \ldots, n_{i}\right\}$

219 The term $m_{i}(t)$ that denotes the true and unobserved value of the longitudinal outcome at 220 time t. Note that $m_{i}(t)$ is different from $y_{i}(t)$, with the latter being the contaminated with 221 measurement error value of the longitudinal outcome at time $t$. To quantify the strength of 222 the association between $m_{i}(t)$ and the risk for an event, a straightforward approach is to 223 postulate a relative risk model of the form:

224

225

226

$$
\left\{\begin{array}{l}
h_{i}\left(t \backslash M_{i}(t), w_{i}\right)=\lim _{d t \rightarrow 0} \operatorname{Pr}\left(\frac{\mathrm{t} \leq T_{i}^{*}<\mathrm{t}+\mathrm{dt} \backslash T_{i}{ }^{*}>\mathrm{t}, M_{i}(t), \mathrm{wi}}{d t}\right) \\
h_{i}\left(t \backslash M_{1 i}(t), w_{i}\right)=h_{0}(\mathrm{t}) \exp \left\{\gamma^{T} W_{i}+\alpha_{1} m_{1 i}(t)\right\}, t>0
\end{array}\right.
$$


228 where $M_{i}(t)=\left\{m_{i}(s), 0 \leq \mathrm{s}<\mathrm{t}\right\}$ denotes the history of the true unobserved longitudinal

229 process up to time point $\mathrm{t}, h_{0}($.$) denotes the baseline risk function and W_{i}$ is a vector of 230 baseline covariates with a corresponding vector of regression coefficients $\gamma$. Similarly, 231 parameter $\alpha_{1}$ quantifies the effect of the underlying longitudinal outcome to the risk for an 232 event

$$
h_{i}(t)=h_{o}(t) \exp \left\{\gamma^{T} w_{i}+\alpha_{1} m_{i}(t)+\alpha_{2} m_{i}^{\prime}(t)\right\}
$$

234

$$
\text { where, } m_{i}^{\prime}(t)=\frac{d}{d_{t}} m_{i}(t)=\frac{d}{d_{t}}\left\{x_{i}^{T}(t) \beta+z_{i}^{T}(t) b_{i}\right\}
$$

$\alpha_{2}$ measures how strongly associated is the value of the slope of the true longitudinal $m_{i}(t)$ remains constant. (23). The relative risk model (1) postulates that the risk for an event at time $t$ depends only on the current value of the time-dependent marker $m_{i}(t)$. The relative risk model (2) postulates that the risk depends on both the current true value of the trajectory and the slope of the true trajectory at time $\mathrm{t}$.

\section{Ethical consideration}

242 Ethical clearance was obtained from the Institutional Review committee of the UOG.

243 Informed consent is not required as the whole data had been retrieved from the medical

244 records of patient. Data anonyms and hold on a secure password protected system.

245 Confidentiality during all phases of research activities was kept. 


\section{Results}

\section{Descriptive Statistics}

251 Among the total of type 2 DM patients during the time period $80(17.17 \%)$ were developed

252 diabetic retinopathy. The mean age of participants at baseline was $53.17(\mathrm{SD}=10.11)$ years 253 and from the total study subjects $69.74 \%$ were within age category $<60$ years. The mean 254 duration of diabetes was 9.2 years $(\mathrm{SD}= \pm 3.8)$. Among $249 \mathrm{~T} 2 \mathrm{DM}$ patients whose baseline 255 FBS was above $200 \mathrm{mg} / \mathrm{dl}, 55 \%$ have developed diabetic retinopathy. Out of a total of 78 256 patients who developed neuropathy $22.5 \%$ have developed diabetic retinopathy (Table 1).

\section{Table 1}

258 To understand the association between the FBS measurement and time individual profile 259 plots were employed. Loess smoothing technique over individual profile plots were used 260 to explore the mean change of FBS measurement over time (Fig 1).

\section{Figure 1: Individual profile plot and the evolution of mean structure of FBS over}

262 time for type 2 diabetes patients in UGCSH, 2001 to 2016

263 As indicated on the plots the individual profile plots suggested that there was within and 264 between variations of change in FBS measurements over time. The individual trajectory of 265 FBS for patients shows that patients had considerably different FBS at baseline and 266 overtime. This suggests a model with both random intercept and slop is a good start. 267 However, the red line which shows the mean structure of FBS measurement over time with 268 loess smoothing technique suggested that the linear change of the mean of FBS 269 measurement over time.

\section{Sensitivity analysis}

271 When FBS were not observed during a 3-month period, values were considered as missing. 272 A problem in the handling of missing data in longitudinal outcomes is the fact that the 
observed data alone cannot distinguish between a MAR and a MNAR dropout mechanism.

274 To handle this, we do sensitivity analysis then analyze the sensitivity of the reported results

275 by observing the parameter estimate and the standard error estimate. The parameter

276 estimate between complete case analysis and multiple imputation is not that much different.

277 So, by considering this joint model with current parametrization with complete case

278 analysis approach was chosen (Table 2).

279 Table 2. Comparison of joint model with Current value and slope parameterization 280 under multiple imputation and complete case analysis missing handling method for 281 T2DM patients.

\begin{tabular}{|c|c|c|}
\hline Method & Parametrization & AIC \\
\hline Multiple imputation & Current & 68944 \\
\cline { 2 - 3 } & Slope & 68830 \\
\hline Complete case analysis & Current & 27625 \\
\cline { 2 - 3 } & Slope & 27719 \\
\hline
\end{tabular}

283 AIC agree that the joint model with the Current parametrization with complete case 284 analysis missing handling approach has a better predictive ability.

\section{Time to Retinopathy}

286 The cumulative survival probability of the patients at the end of 12,36 and 72 months were $28797 \%, 94 \%$, and 89\%, respectively. The cohort contributed a total of 3607.67 person-years. 288 About half (51\%) diabetic retinopathy were developed with in the first five years. The 289 median follow up time was $90.8[\mathrm{IQR}=63.4]$ months and a total of $80(17.17 \%)$ patients 290 developed DR. The incidence density was 2 cases per 100 patients per year (95\%CI: 1.78 
291 2.76). The median survival time or the survival time at which the cumulative survival

292 function is equals to 0.5 could not be determined (Fig 2).

293 Figure 2: The plot of the overall estimate of Kaplan-Meier survivor for time to

294 diabetic retinopathy among T2DM patients under anti diabetes treatment at

295 UGCSH, 2001-2016

296

297

298

299

300

301

302

303

304

305 (Diabetic retinopathy).

306

307

308

309

310

311 diabetic retinopathy (Table 3).

312 The hazard of diabetic retinopathy for hypertensive patient is $2.49[1.324 .66]$ times higher

313 than those patients who are not hypertensive keeping other variables constant.

314 Unexpectedly, the hazard of developing DR among patient's duration 6-10 years is 69\% 
315 less likely than patient's duration less than 6 years keeping other variables constant. The 316 hazard of developing DR among patient's duration $>10$ years is $83 \%$ less likely than

317 patient's duration less than 6 years keeping other variables constant.

318 The hazard of developing DR for patients who take more than one oral anti diabetic agents 319 together to manage their diabetes is $4.90[1.0720 .0]$ times higher than those patients who 320 are not taking any medication keeping other variables constant

321 A unit increase in the current value of fasting blood sugar increase the hazard of developing 322 diabetic retinopathy by $1.35[95 \% \mathrm{CI}: 1.121 .63]$ times, whereas a unit increase in the rate of 323 fasting blood sugar trajectory increase the hazard of diabetic retinopathy by $1.70[95 \%$ CI: $3241.21 ; 2.39$ ] times, provided that the true an observed value remain constant (Table 3).

325 The joint model could be fitted like $H_{i}\left[t \mid M_{i}(t), W_{i}\right]$ $=0.91 *$ hypertension $-0.18 *$ femalesex $-0.20 *$ baselineage $-1.16 *$ duration $_{6-10}-1.75 *$ duration

$$
>10 \text { year }+1.93 * 1 \text { oral agent }+1.59 *
$$

$$
+0.37 * B M I_{25-29.9}+0.14 * B M I_{\geq 30}+0.30 * M_{i}(t)
$$

332

Where, $M_{i}(t)$ is the true unobserved longitudinal process up to time t. $W_{i}$ is the vector of fixed effect covariates.

334 Table 3. The fitted joint model result under current parameterizations for type 2 diabetes patients in UGCSH ,2001 - 2016.

\begin{tabular}{|l|l|l|l|l|l|}
\hline \multicolumn{2}{|l|}{ Longitudinal sub model } & \multicolumn{3}{l|}{ Survival sub model } \\
\hline Fixed effects & Coeff $(\beta)$ & 95\% CI & Covariates & AHR & 95\% CI \\
\hline & & & Sex & & \\
\hline Intercept & 13.50 & {$[11.4615 .55] * * *$} & Male & 1 & \\
\hline
\end{tabular}




\begin{tabular}{|c|c|c|c|c|c|}
\hline FBS time 1 & -0.288 & {$\left[\begin{array}{lll}-0.93 & 0.35\end{array}\right]$} & Female & 0.83 & {$\left[\begin{array}{lll}0.43 & 1.59\end{array}\right]$} \\
\hline FBS time 2 & -4.013 & {$[-4.70-3.32] * * *$} & HTN & & \\
\hline FBS time 3 & -1.444 & {$[-1.92-0.96] * * *$} & No & 1 & \\
\hline Baseline SBP & & & Yes & 2.49 & {$[1.324 .66] * *$} \\
\hline$\leq 140$ & 0 & & Medication & & \\
\hline$>140$ & 0.045 & {$\left[\begin{array}{lll}-0.51 & 0.60\end{array}\right]$} & No medication & 1 & \\
\hline Current Age & -0.255 & {$[-0.49-0.02] * * *$} & 1 oral agent & 2.55 & [0.43 7.38] \\
\hline Medication & & & $>1$ oral agent & 4.90 & {$[1.0720 .0] *$} \\
\hline Dietary modification & 0 & & Insulin & 4.28 & {$\left[\begin{array}{lll}0.78 & 21.2\end{array}\right]$} \\
\hline 1 oral agent & 0.547 & {$\left[\begin{array}{lll}-0.32 & 1.41]\end{array}\right.$} & Neuropathy & & \\
\hline$>1$ oral agent & 0.902 & {$\left[\begin{array}{lll}0.21 & 1.59\end{array}\right] * *$} & No & 1 & \\
\hline Insulin & 0.701 & {$\left[\begin{array}{lll}-0.18 & 1.58\end{array}\right]$} & Yes & 1.76 & {$\left[\begin{array}{lll}0.81 & 3.81\end{array}\right]$} \\
\hline Sex & & & Nephropathy & & \\
\hline Male & 0 & & No & 1 & \\
\hline Female & 0.130 & {$\left[\begin{array}{lll}-0.35 & 0.61]\end{array}\right.$} & Yes & 1.47 & {$\left[\begin{array}{lll}0.63 & 3.42\end{array}\right]$} \\
\hline Duration & 0.096 & {$\left[\begin{array}{lll}0.03 & 0.16\end{array}\right] * * *$} & Duration & & \\
\hline Weight & 0.034 & {$[-0.05-0.02] * * *$} & $\leq 5.9$ years & 1 & \\
\hline \multicolumn{3}{|c|}{ Variance Component } & 6-10 years & 0.31 & {$\left[\begin{array}{lll}0.11 & 0.84\end{array}\right] *$} \\
\hline Std. Dev. (intercept) & \multicolumn{2}{|c|}{1.66} & $\geq 10$ years & 0.17 & {$\left[\begin{array}{llll}0.06 & 0.46\end{array} * * *\right.$} \\
\hline Std. Dev. (Time) 1 & \multicolumn{2}{|c|}{3.86} & \multicolumn{3}{|c|}{ Association parameters } \\
\hline Std. Dev. (Time)2 & \multicolumn{2}{|c|}{2.41} & Parametrization & & \\
\hline Std. Dev. (Time)3 & \multicolumn{2}{|c|}{0.86} & Current & 1.35 & {$\left[\begin{array}{lll}1.12 & 1.63\end{array}\right]^{* * *}$} \\
\hline Std. Dev. (Residual) & \multicolumn{2}{|c|}{3.17} & Slope & 1.70 & {$\left[\begin{array}{lll}1.21 & 2.39\end{array}\right] * * *$} \\
\hline
\end{tabular}

\section{Discussion}

338 Diabetic retinopathy is a long-term complication of diabetes mellitus that could be

339 attributed to systemic or local ocular factors (24). The disease is asymptomatic until it leads

340 to visual deterioration. Monitoring the biomarkers may be essential to be aware of the

341 factors which increase the likelihood of retinopathy and to act accordingly. Joint modeling 
approach was applied in order to determine how strong is the association between fasting blood sugar and the risk of diabetic retinopathy.

344 Nearly half of diabetic retinopathy were developed with in the first five years. This is 345 consistent with the study done in Arbaminch (14) which shows that $47 \%$ of diabetic 346 retinopathy developed with in the first five years. This could be due to most of type 2 DM 347 patients came to the health center when they face any complication including visual 348 problem since it is asymptomatic until the advanced stage. However, this study finding is 349 higher than the study done in Pakistan (25) which showed that only 22\% of diabetic retinopathy developed with in the first five years.

351 The results of this study show that the proportion of DR was $17.17 \%$ with 20 cases per 3521000 year. The study showed a higher incidence of DR than studies done in Ethiopia and 353 China $(21,26)$. This inconsistency might be due to the difference in health care service and 354 complication monitoring. However, the incidence rate in this study was lower than that of 355 a study done at Arbaminch general hospital, Ethiopia (14) and retrospective longitudinal 356 follow-up study done in Pakistan (25). The difference in study period, diagnostic methods 357 used by the studies and the denominator population might have attributed to this difference. 358 The current study showed that duration of diabetes was negatively associated with hazard 359 of DR and it is consistent with longitudinal study finding on diabetic patients in Arbaminch 360 (14) but it is not consistent with longitudinal study finding on diabetic patients in Malaysia 361 (27) and cross-sectional study finding in Jimma university tertiary hospital and tertiary care 362 hospital in Pakistan $(15,28)$. This might be since our study populations were type 2 DM 363 patients who are more likely to come late to the health facility and seek health services 364 because the disease process of T2DM is more gradual and not as severe as type 1 DM. 
365 Another possible explanation for this finding, type 2 DM patients may gradually develop a better metabolic control with low levels of FBS.

367 The result revealed that hypertension is a risk factors for diabetic retinopathy. this finding 368 is consistent with the studies from Ethiopia $(14,29)$ and also by UK Prospective Diabetes 369 Study (UKPDS 38) (30). Increased blood flow could damage the retinal capillary 370 endothelial cells in eyes of people with diabetes (31). The pathophysiological mechanism

371 involves the function and interaction of the endothelial and vascular smooth muscle cells.

372 damage and altered growth of retinal capillary endothelial cells induce proliferative lesions 373 of the retina (32).

374 The results showed that taking more than one oral anti diabetic agents together to manage 375 their diabetes increased the risk of diabetic retinopathy. This might be due to combination 376 of oral agent is generally advocated when no medication or only one oral medication users 377 had poorly control glycemia at some points and when patients develop resistance to 378 medication which is expressed by poor glycemic control. Prolonged poor glycemia control 379 causes injuries to the retinal vasculature. But other studies $(14,21,27)$ shows that 380 medication has no association with risk of diabetic retinopathy. So further investigation is 381 needed.

382 In this study, we observe strong evidence that both current value of FBS and rates of FBS 383 change were associated with diabetic retinopathy. Several earlier studies on type 2 DM 384 patients showed that fasting blood sugar level is significantly associated with diabetic 385 retinopathy $(14,16,26,33)$. But all these studies could not able to identify the effects of 386 longitudinal FBS trajectory on the risk diabetic retinopathy. By using joint modelling 387 approach, we estimate the effects of longitudinal trajectory rate on the risk of DR. 
388 One of the strengths of this study was the accuracy of diabetic retinopathy since grading

389 is not only clinical ophthalmologic examination but also fundus photographs.

390 The main limitation of this study was data on some potentially important predictors were 391 not available which may underestimate the effects and individual variations in the 392 development of diabetes retinopathy (some sociodemographic factors and behavioral 393 factors). Another limitation was in our study area HBA1C were not done for follow-up

394 which is the recommended mode of testing blood sugar control, leading to the use of fasting 395 blood sugar alone.

\section{Conclusion}

397 This study revealed that type of medication, duration, weight, current age, and time were 398 found significant predictors of change in fasting blood sugar, whereas presence of 399 hypertension, medication and duration were significant predictors of time to diabetic 400 retinopathy. Unobserved true current value of FBS and rate of FBS change were 401 significantly associated with time to diabetic retinopathy. Based on the study finding from 402 this data we recommend that, it is better to do screening for diabetic retinopathy at the time 403 of diagnosis of DM since most diabetes patients develop diabetic retinopathy at the early 404 period of the follow up and to give special attention for those who took combined 405 medication in the screening program. The health care provider strength the routine 406 monitoring of FBS as a biomarker which provides meaningful prognostic information that 407 can help to differentiate patients with regard DR and lead the way to change other 408 intervention and close monitoring of patients diagnosed with hypertension.

\section{Abbreviation}

410 AHR: Adjusted Hazard Ratio;

411 AIC: Akaike Information Criteria; 
BMI: Body Mass Index;

413 DBP: Diastolic Blood Pressure;

414 DM: Diabetes Mellitus;

415 DR: Diabetic Retinopathy;

416 FBS: Fasting Blood Sugar;

417 IDF: International Diabetic Federation;

418 IQR: Inter Quartile Range;

419 SBP: Systolic Blood Pressure;

420 T2DM: Type Two Diabetes Mellitus;

421 UGCSH: University of Gondar Comprehensive Specialized Hospital;

422 WHO: World Health Organization

423 Declarations

424 Ethics approval and consent to participate

425 Ethical clearance was obtained from the Institutional Review committee of the University

426 of Gondar.

427 Consent for publication

428 Not applicable

429 Availability of data and materials

430 All necessary information's were included with in the manuscript

431 Competing interests 
The authors declare that they have no competing interests

\section{Funding}

434 The data collection was financially covered by university of Gondar.

435 Authors' Contributions

436 Conception of the work, design of the work, acquisition of data, analysis and interpretation 437 of data was done by SA. Data curation, drafting the article, revising it critically for 438 intellectual content, validation and final approval of the version to be published was done 439 by SA, ZT, SA and TA. All authors read and approved the final manuscript.

\section{Acknowledgments}

441 We thank University of Gondar for giving ethical clearance to conduct this research. Our 442 gratitude also goes to supervisors and data collectors who actively participated in this 443 research work.

\section{Reference}

445 1.WHO. World Health Organization. Fact Sheet No.312: What is Diabetes?

446 2.WHO. Definition, diagnosis and classification of diabetes mellitus and its complications:

447 report of a WHO consultation. Part 1, Diagnosis and classification of diabetes mellitus.

448 Geneva: World health organization, 1999.

449 3.ADA. Classification and diagnosis of diabetes: standards of medical care in diabetes450 2018. Diabetes care. 2018;41(Supplement 1):S13-S27.

451 4.Goldenberg R, Punthakee Z. Definition, classification and diagnosis of diabetes, 452 prediabetes and metabolic syndrome. Canadian journal of diabetes. 2013;37:S8-S11.

453 5.IDF.International Diabetes Federation. ATLAS.2017 ;Eighth edition

454 6.Communicable Disease Control .Type 2 Diabets.Downloaded from CDC at 455 https://www.cdc.gov/diabetes/basics/type2.html (accessed on 26/1/2019). 
7.Sayin N, Kara N, Pekel G. Ocular complications of diabetes mellitus. World journal of

457 diabetes. 2015;6(1):92.

8.ADA.Diagnosis and classification of diabetes mellitus. Diabetes care. 2014;37(Supplement 1):S81-S90.

460 9.Zheng Y, He M, Congdon N. The worldwide epidemic of diabetic retinopathy. Indian 461 journal of ophthalmology. 2012;60(5):428.

462 10.Burgess P, MacCormick I, Harding S, Bastawrous A, Beare N, Garner P. Epidemiology 463 of diabetic retinopathy and maculopathy in Africa: a systematic review. Diabetic medicine. $464 \quad 2013 ; 30(4): 399-412$.

465 11.Mathenge W, Bastawrous A, Peto T, Leung I, Yorston D, Foster A, et al. Prevalence 466 and correlates of diabetic retinopathy in a population-based survey of older people in 467 Nakuru, Kenya. Ophthalmic epidemiology. 2014;21(3):169-77.

468 12.Abebe N, Kebede T, Addise D. Diabetes in Ethiopia 2000-2016-prevalence and related 469 acute and chronic complications; a systematic review. African Journal of Diabetes 470 Medicine. 2017;25(2).

471 13.Solomon SD, Chew E, Duh EJ, Sobrin L, Sun JK, VanderBeek BL, et al. Diabetic 472 retinopathy: a position statement by the American Diabetes Association. Diabetes care. $473 \quad 2017 ; 40(3): 412-8$.

474 14.Chisha $\mathrm{Y}$, Terefe $\mathrm{W}$, Assefa H. Incidence and factors associated with diabetic 475 retinopathy among diabetic patients at arbaminch general hospital, gamo gofa Zone 476 (longitudinal follow up data analysis). Journal of Diabetology. 2017;8(1):1.

477 15.Hussain S, Qamar MR, Iqbal MA, Ahmad A, Ullah E. Risk factors of retinopathy in 478 type 2 diabetes mellitus at a tertiary care hospital, Bahawalpur Pakistan. Pakistan journal 479 of medical sciences. 2013;29(2):536. 
481 Patients in Benin HSOA Journal of Ophthalmology and Clinical Research. 2014.

483 among adults in an urban community in Tanzania: University of Otago; 2010.

484 18.international Diabetes Federation 2019. eight edition:

485 19.Diggle P, Diggle PJ, Heagerty P, Heagerty PJ, Liang K-Y, Zeger S. Analysis of 486 longitudinal data: Oxford University Press; 2002.

487 20.Moges WK, Muralidharan A, Tadesse HZ. Gaussian Longitudinal Analysis of 488 Progression of Diabetes Mellitus Patients Using Fasting Blood Sugar Level: A Case of 489 Debre Berhan Referral Hospital, Ethiopia. American Journal of Theoretical and Applied 490 Statistics. 2018;7(1):21-8.

491 21.Azeze TK, Sisay MM, Zeleke EG. Incidence of diabetes retinopathy and determinants 492 of time to diabetes retinopathy among diabetes patients at Tikur Anbessa Hospital, 493 Ethiopia: a retrospective follow up study. BMC research notes. 2018;11(1):542.

494 22.Dodson P. Diabetic Retinopathy (Oxford Diabetes Library)Screening to Treatment: 495 Oxford University Press; 2011 2011-05.

496 23.Rizopoulos D. Joint models for longitudinal and time-to-event data: With applications 497 in R: Chapman and Hall/CRC; 2012.

498 24.Frank RN. Diabetic retinopathy and systemic factors. Middle East African journal of 499 ophthalmology. 2015;22(2):151.

500 25.Asher Fawwad NM, Zafar AB, Khalid M. Incidence of microvascular complications of 501 type 2 diabetes: A 12 year longitudinal study from Karachi-Pakistan. Pakistan journal of 502 medical sciences. 2018;34(5):1058. 
504 diabetic retinopathy within type 2 diabetes: a five-year cohort study in China (report 1).

505 International journal of environmental research and public health. 2015;12(7):7899-909.

506 27.Abougalambou SSI, Abougalambou AS. Risk factors associated with diabetic 507 retinopathy among type 2 diabetes patients at teaching hospital in Malaysia. Diabetes \&

508 Metabolic Syndrome: Clinical Research \& Reviews. 2015;9(2):98-103.

509 28.Worku D, Hamza L, Woldemichael K. Patterns of diabetic complications at jimma 510 university specialized hospital, southwest ethiopia. Ethiopian journal of health sciences. $5112010 ; 20(1)$

512 29.Wolde HF, Atsedeweyen A, Jember A, Awoke T, Mequanent M, Tsegaye AT, et al.

513 Predictors of vascular complications among type 2 diabetes mellitus patients at University

514 of Gondar Referral Hospital: a retrospective follow-up study. BMC endocrine disorders.

$515 \quad 2018 ; 18(1): 52$.

516 30.Group UPDS. Tight blood pressure control and risk of macrovascular and microvascular

517 complications in type 2 diabetes: UKPDS 38. BMJ: British Medical Journal. 518 1998;317(7160):703.

519 31.Klein R. Diabetic retinopathy and nephropathy. The diabetic kidney: Springer; 2006. 520 p. 473-98.

521 32.Hsueh WA, Anderson PW. Hypertension, the endothelial cell, and the vascular 522 complications of diabetes mellitus. Hypertension. 1992;20(2):253-63.

523 33.Massin P, Lange C, Tichet J, Vol S, Erginay A, Cailleau M, et al. Hemoglobin A1c and 524 fasting plasma glucose levels as predictors of retinopathy at 10 years: the French DESIR 525 study. Archives of Ophthalmology. 2011;129(2):188-95.

\section{$526 \quad$ Figure legends}


527 Figure 1: The individual profile plots suggested that there was within and between 528 variations of change in FBS measurements over time. The individual trajectory of FBS for 529 patients shows that patients had considerably different FBS at baseline and overtime. This 530 suggests a model with both random intercept and random slop is required. The red line

531 (loess smoothing technique) which shows the mean structure of FBS measurement over 532 time suggested that the linear change of the mean of FBS measurement over time. FBS, 533 Fasting Blood Sugar

534 Figure 2: The plot of the overall estimate of Kaplan-Meier survivor for time to diabetic 535 retinopathy among T2DM patients under anti diabetes treatment at UGCSH, 2001-2016. 536 Black line indicated the survival estimate, the red and green color line show the lower and 537 the upper boundary of the estimate. The median survival time or the survival time at which 538 the cumulative survival function is equals to 0.5 could not be determined

539 Table 1. Demographic and Clinical characteristics of the study participants in

540 UGCSH, 2001-2016. Exploring fasting blood sugar change level

\begin{tabular}{|c|c|c|c|}
\hline Variable & Censored (\%) & $\begin{array}{l}\text { Diabetic } \\
\text { Retinopat }\end{array}$ & Total \\
\hline \multicolumn{4}{|l|}{ Sex } \\
\hline Male & $153(39.64)$ & $34(42.50)$ & $187(40.13)$ \\
\hline Female & $233(60.36)$ & $46(57.50)$ & $279(59.87)$ \\
\hline Baseline Age & \multicolumn{2}{|c|}{ Mean $=53.17 \pm 10.11$} & \\
\hline$<60$ & $266(68.91)$ & $59(73.75)$ & $325(69.74)$ \\
\hline$\geq 60$ & $120(31.09)$ & $21(26.25)$ & 141(30.26) \\
\hline \multicolumn{4}{|l|}{ Residence } \\
\hline Gondar & $309(80.05)$ & $69(86.25)$ & $378(81.12)$ \\
\hline
\end{tabular}


Out of Gondar $\quad$ 77(19.95) $\quad$ 11(13.75) $\quad$ 88(18.88)

Baseline FBS $\quad$ Mean $=224.3 \pm 81.76$

$70-130$

$33(8.55)$

$2(2.50)$

35

$131-200$

$148(38.34)$

$34(42.50)$

182

$>200$

205(53.11)

$44(55)$

249

HTN

Yes

79(20.49)

29(36.25)

108

No

307(79.53)

51(63.75)

358

Medication

$\begin{array}{llll}\text { No medication } & 25(6.48) & 4(6.25) & 29 \\ \text { 1 oral agent } & 247(63.9) & 51(62.5) & 298 \\ >1 \text { oral agent } & 68(17.6) & 12(15) & 80 \\ \text { Insulin } & 46(11.9) & 13(16.3) & 59\end{array}$

Neuropathy

Yes

60(15.5)

18(22.5)

78

No

326(84.5)

62(77.5)

388

Nephropathy

Yes

55(14.25)

52(15)

67

No

331(85.75)

28(85)

399

Anemia

Yes

31(8.03)

7(8.75)

38

No

355(91.97)

73(91.25)

428

Duration

Mean $=9.2 \pm 7.7$

$<6 \mathrm{yr}$

82(21.2)

18(22.5)

100

6-10 yr

174(45.08)

31(38.75)

205 
$\begin{array}{llll}>10 \mathrm{yr} & 130(33.68) & 31(38.75) & 161\end{array}$

Baseline SBP

$\begin{array}{llll}\leq 140 & 308(79.79) & 69(86.25) & 377\end{array}$

$\begin{array}{llll}>140 & 78(20.21) & 11(13.75) & 89\end{array}$

Baseline DBP

$\begin{array}{llll}\leq 90 & 344(89.12) & 72(90) & 416\end{array}$

$\begin{array}{llll}>90 & 42(10.88) & 8(10) & 50\end{array}$

541

542

543

544

545

546

547 


\section{Figures}

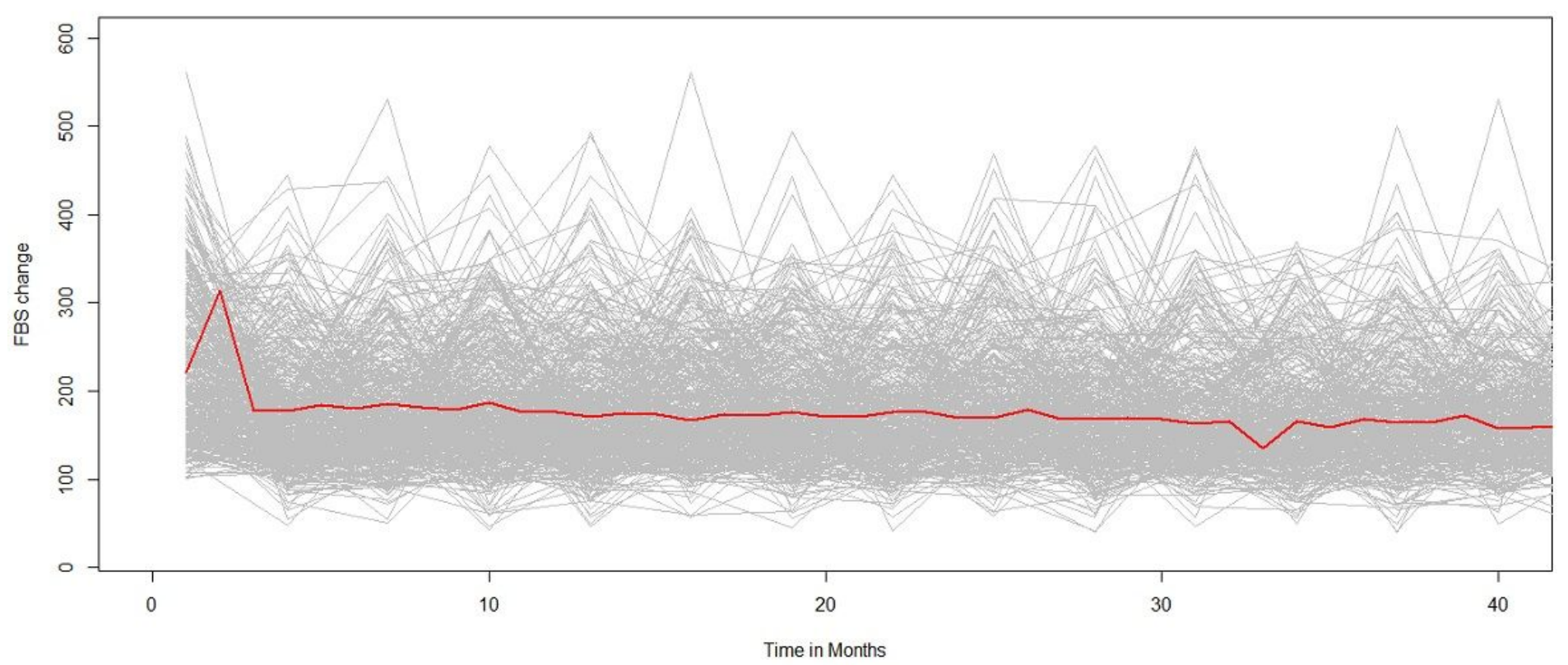

Figure 1

Individual profile plot and the evolution of mean structure of FBS over time for type 2 diabetes patients in UGCSH, 2001 to 2016. The individual profile plots suggested that there was within and between variations of change in FBS measurements over time. The individual trajectory of FBS for patients shows that patients had considerably different FBS at baseline and overtime. This suggests a model with both random intercept and random slop is required. The red line (loess smoothing technique) which shows the mean structure of FBS measurement over time suggested that the linear change of the mean of FBS measurement over time. FBS, Fasting Blood Sugar 


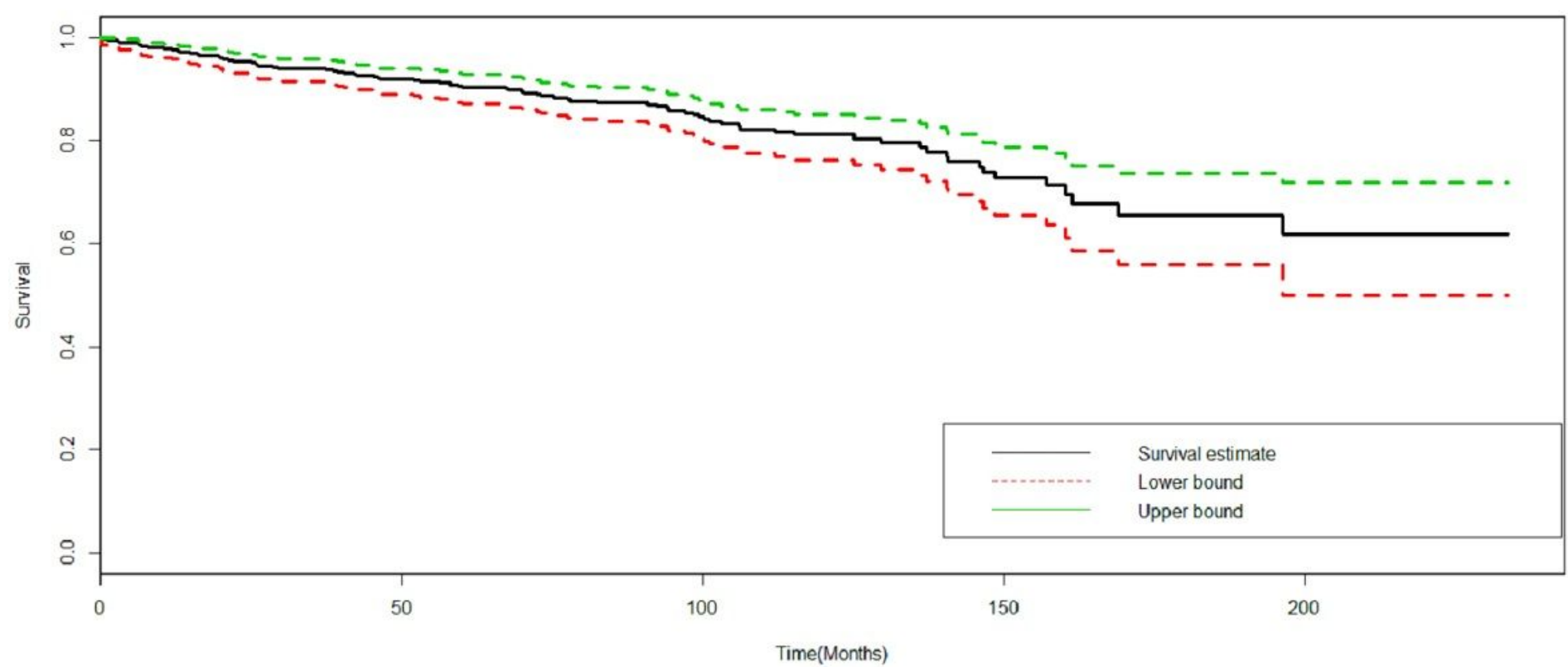

\section{Figure 2}

The plot of the overall estimate of Kaplan-Meier survivor for time to diabetic retinopathy among T2DM patients under anti diabetes treatment at UGCSH, 2001-2016. The plot of the overall estimate of KaplanMeier survivor for time to diabetic retinopathy among T2DM patients under anti diabetes treatment at UGCSH, 2001-2016. Black line indicated the survival estimate, the red and green color line show the lower and the upper boundary of the estimate. The median survival time or the survival time at which the cumulative survival function is equals to 0.5 could not be determined 